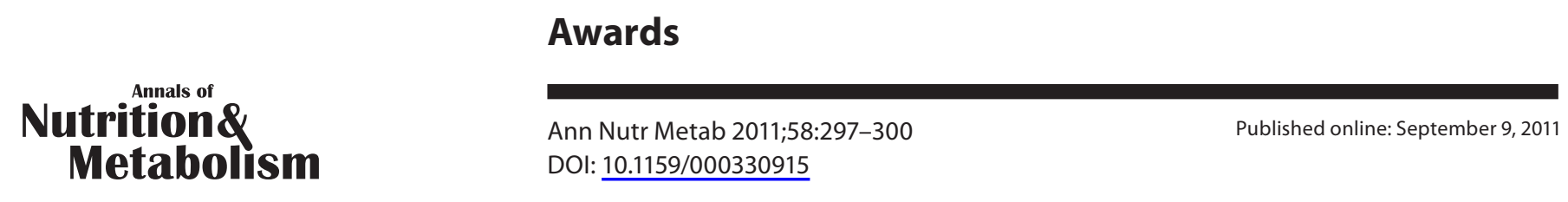

\title{
DSM Science \& Technology Awards (South) Event 2011: The Role of Micronutrients in Human Nutrition
}

\begin{abstract}
On 21 June 2011, the DSM Science \& Technology Awards recognized and rewarded outstanding innovative young scientists active in fundamental and applied research. As in previous years, an international judging committee honored creative and groundbreaking $\mathrm{PhD}$ research. Twelve finalists, the top candidates from Switzerland, Austria, France and southern Germany, were invited for the final competition followed by the award ceremony attended by numerous representatives of universities, non-governmental organizations, institutes of technology and industry.
\end{abstract}

Copyright $\odot 2011$ S. Karger AG, Base

\section{Introduction}

The DSM Science \& Technology Awards seek to recognize and reward outstanding young researchers who are conducting innovative research with clear potential for practical applications. The awards are open to researchers working in the fields of advanced synthetic methods (chemistry and catalysis), nutrition science, food science, process design and process technology, biotechnology, materials chemistry (chemistry of polymers and resins), as well as materials properties and processing. Two international European regions are covered in these awards - the northern region (comprising of the Netherlands, Belgium and northern Germany) and the southern region (Switzerland, Austria, north-eastern France and southern Germany). For the southern region, twelve awards in total go out every year to promising researchers. In recognition of their achievement, all twelve finalists receive a certificate and a cash prize. On 21 June 2011, the ceremony for the awards (south) took place at the Grand Hotel Victoria-Jungfrau, Interlaken, Switzerland.

\section{Report}

The awards were presented by Manfred Eggersdorfer, Chairman International Jury and Senior Vice President Nutrition Science and Advocacy, DSM Nutritional Products (DNP). In his welcome speech Mr. Eggerdorfer addressed the role of basic science as a catalyst for innovation. Basic science is essential and forms the basis for the close link that exists between scientific research and application research in industry. DSM's motivation for creating the Science \& Technology Awards was to support and spur on this important interrelation. The scientific dialog between researchers at universities and industry, plus the resulting network, aids in the development of solutions to current societal challenges. In today's world, there are many problems that require new scientific insights and breakthroughs. One of the most important issues is adequate nutrition and nutrition security for all.

\section{KARGER}

Fax +4161306 1234

E-Mail karger@karger.ch

www.karger.com
(C) 2011 S. Karger AG, Base

0250-6807/11/0584-0297\$38.00/0

Accessible online at:

www.karger.com/anm 
In particular, research into the role of micronutrients in human nutrition to support health and healthy aging requires joint efforts by all stakeholders from academia, governmental and non-governmental organizations, as well as industry. At present, more than 3 billion people suffer from malnutrition; an estimated 925 million people worldwide suffer from starvation with 17.5 million deaths, and 18 million babies born mentally impaired per year due to malnutrition. Moreover, inadequate micronutrient intake and status is not only an issue in poor countries, it is also a topic in industrialized countries, as demonstrated by food intake surveys. In addition, global megatrends, such as aging populations and urbanization, coupled with lifestyle-associated unbalanced nutrition (e.g. fast food and the trend towards processed foods) make inadequate micronutrient intakes a global problem. Micronutrient deficiencies and insufficiencies have been shown to exacerbate the development of many chronic health disorders, such as cardiovascular and metabolic diseases, cancer and osteoporosis. Addressing these various challenges, the awards began with a series of presentations, starting with the business focus, continuing onto a case study on vitamin E, and summing up with the future development of health care costs, the role of healthy nutrition and the impact of the latest scientific discoveries.

Gareth Barker, Head Global Marketing Human Nutrition at DNP, noted that over the last decade a renaissance has taken place for vitamins. New studies showing the short- and long-term effects of inadequate micronutrient intakes for health, innovative technologies (e.g. analytics and biomarkers, micronutrient-gene interactions) and emerging new health benefits have resulted in a re-evaluation of what adequate intake means. The groups that are in jeopardy of not consuming enough vitamins tend to be the same the world over: no matter where they are, school-aged children and teenagers run the risk of having inadequate intakes of vitamins A, C, D, E and folate due to nutrient-poor diets, rapid growth spurts, dieting for weight loss and unbalanced food patterns. Elderly people are more likely to be deficient in vitamins $\mathrm{A}, \mathrm{E}, \mathrm{D}, \mathrm{C}, \mathrm{B}_{1}$, $B_{2}, B_{3}, B_{12}$ and folate because of reduced intestinal absorption, decreased ability to synthesize certain vitamins (such as vitamin D), use of multiple medications or physical disability. In addition to that, studies have shown that many pregnant women, who have higher nutritional demands, have dietary gaps for several nutrients. For the general abatement of perilous cases, Barker highlighted vitamin $\mathrm{D}$ as an example of one among many with newly discovered potential for disease risk reduction. The re- cently discovered health benefits of vitamin $\mathrm{D}_{3}$ go far beyond bone health to include muscle health as well as the potential to boost the cardiovascular, metabolic and immune systems. However, awareness of micronutrient deficiency is striking: $50 \%$ of the adult European population is vitamin $\mathrm{D}$ deficient with blood concentrations below $50 \mathrm{nmol} / 1$ [1]. Consequently, the Institute of Medicine tripled the dietary reference intakes for vitamin $\mathrm{D}_{3}$ from 200 to 600 IU per day in 2010.

In their dual presentation Peter Weber, Corporate Scientist, DNP, and Peter Schuler, Technical Marketing Human Nutrition \& Health, DNP, elucidated a case study on a vitamin that plays a key role in human nutrition and health: vitamin E, $\alpha$-tocopherol being the predominant form in the human body. This vitamin is an important antioxidant, which has been confirmed by the recently issued European Food Safety Authority health claim. The evidence taken all together clearly supports the role of vitamin $\mathrm{E}$ in as a factor in diminishing the risk of cardiovascular disease [2]. However, the results from randomized controlled trials have not detected consistent beneficial results from vitamin E supplementation on cardiovascular health [3]. Research has provided an insight into this apparent discrepancy: in several human intervention studies investigating the effect of vitamin $\mathrm{E}$ on cardiovascular health, the vitamin E serum level in the placebo group was already close to levels defined as beneficial. Due to a ceiling effect, no further disease-risk-reducing effect could be expected by increasing this vitamin E level by supplementation. In addition, individuals with a specific genetic make-up, who account for a third of the European population, seem to benefit from vitamin E more than others. For instance, diabetics carry a particular polymorphism in the haptoglobin gene, coding for a protein that scavenges free hemoglobin in the blood. $\mathrm{Hu}-$ man studies show their cardiovascular systems benefit from a daily dose of $400 \mathrm{IU}$ vitamin $\mathrm{E}$ [4]. The underlying mechanism of action is assumed to be related to the antioxidative function of vitamin E, which may especially benefit diabetics who suffer from increased oxidative stress. From epidemiological studies, vitamin E serum levels of $30 \mu \mathrm{mol} / \mathrm{l}$ have been conjectured to reduce the risk of cardiovascular events. Published data reveals that a daily intake of ca $33 \mathrm{mg}$ (ca $50 \mathrm{IU}$ ) vitamin E would be required to achieve this vitamin $\mathrm{E}$ serum level [5]. However, current daily intake in the US is only approximately $5 \mathrm{mg}$ on average, leaving more than $90 \%$ of the North Americans with intakes below current recommendations [6]. Vitamin $\mathrm{E}$ in fortified foods and dietary supplements could bridge the vitamin gap here. 
Stephan Tanda, DSM Managing Board Member, emphasized that we live in a century of innovations. The pace of innovation is faster than ever before and breakthroughs in science are key for the challenges ahead - in a world with a population that will grow to 9 or 10 billion people over the coming decades. This requires new approaches for the global challenges in climate and energy, in health and wellness. DSM, the largest vitamin producer in the world, is a science-based company. Scientific research is the basis upon which to create solutions to the big challenges facing our global society - to create innovations that make a positive and sustainable difference to the lives of people around the world. DSM builds on science and believes that the combination of science and application is the cornerstone for innovation. It needs both fundamental academic research and application-oriented industrial research. As a consequence, DSM closely interacts with the academic world, an essential component of our success. DSM recognizes and rewards exceptional achievements in science - both by its own employees and by talents working outside DSM. That is why the company has set up the Innovation Awards Program. During the past quarter-century, more than 20 prize winners have opted for a career at DSM, and some 20 further prize winners have meanwhile become professors at universities.

In his keynote lecture, Stefan Felder, Extraordinarius and Professor for Health Economics, University of Basel, addressed the relationship between aging, health and health care [7]. The population in the developed world has experienced a significant increase in life expectancy over the last 50 years. Simultaneously, while the onset of comorbidities has been deferred to older age groups, health care expenditure has grown dramatically, primarily owing to the advancement of medical technology and the expansion of individual income levels, along with population aging in the wake of increased longevity. However, how population aging contributes to health expenditure increases is the subject of theoretical and empirical scrutiny. An aging population is often blamed for the constant increase in health care expenditure over time. However, it is not age per se but proximity to death that determines the bulk of expenditure; therefore, a shift in the mortality rates to higher ages will not affect lifetime health care expenditures, as death occurs only once in every life. Estimates suggest that longevity has increased by 4.5 years since 1980 , which corresponds to about $13.5 \%$ of life expectancy of a 20 -year-old in 2000 . Furthermore, population aging seems to account for only $0.5 \%$ of the annual growth rate of health care costs. These gains in longevity lead to a rise in demand for life-prolonging medical care. Other possible causes, such as supply-induced demand, moral hazard or the high labor share of health care technology also cannot explain the sharp rise in expenditure. It is the steady increase in income along with progress in medicine and nutrition technology that contributes most to the rising share of health care costs as a segment of GDP in modern societies. Estimates indicate that health care expenditures of modern societies (OECD countries) will reach $40 \%$ of GDP in 2040.

At the conclusion, Manfred Eggersdorfer announced the 1st, 2 nd and 3rd place winners of the DSM Science \& Technology Awards (South) 2011. In her lecture, the 1st prize laureate, Armelle Copet from the Institut Curie CNRS - University Pierre and Marie Curie, addressed the role of the human histone chaperons Asfla and Asflb in the maintenance of genome integrity. In the nucleus of eukaryotic organisms, genomic DNA is packaged into chromatin, a complex nucleoprotein structure. The basic unit of chromatin is the nucleosome, which consists of about 147 bp of DNA wrapped around an octamer of small proteins called histones. This complex organization not only allows for the compaction of DNA, but also carries an extra layer of information crucial to regulating genome function. Corpet focused on a particular protein involved in the dynamics of histones: the histone chaperone Asf1 (anti-silencing function 1). At a fundamental level, she showed that Asf1 participates in the re-organization of DNA into chromatin during its duplication, and thus plays an essential role in cell divisions. In addition, she demonstrated that Asf1 represents a new proliferation marker, which is relevant both for the diagnosis and the prognosis in breast cancer and is potentially a new target for drug discovery in breast cancer [8]. Besides offering new insights, this research opens up the possibility of using new diagnostic and prognostic biomarkers to assess human health and chronic diseases.

In his research, the 2 nd prize laureate, Stefan Radl from the Institute for Process and Particle Engineering at the Graz University of Technology, focused on the theoretical and computational investigation of chemical reactors, aiming to predict the product distribution that results from complex reaction networks in such multiphase reactors. In these reactors, two or more phases (such as gas and liquid) are present, and chemical reactions are entangled with mass transfer processes. A better understanding of this complex interaction of flow and chemical reactions is important, as it aids in promoting the formation of the desired product. This is key for the cost-effi- 
cient and environmentally friendly production of pharmaceuticals, for example, as well as in designing new production processes for increasingly complex chemicals [9]. The innovative aspect of Radl's research is the integration of detailed flow studies to quantify the impact of mixing on the reaction outcome, enabling him to reveal new effects in chemical reactors that cannot be described with current theoretical models.

Finally, the 3rd prize winner, Antoine Diguet from the Department of Chemistry at the Ecole Normale Supérieure Paris, presented his research on the design and implementation of new photosensitive molecules for converting light into biochemical or physical function. At the molecu- lar level, he has developed a system using light to control the organization of DNA as well as in vitro gene expression (protein synthesis). This method is now being applied to develop drug delivery systems that can be selectively activated by an external light stimulus, where and when it is necessary [10]. At the micro- and macroscopic levels, photosensitive molecules for the direct conversion of light energy into mechanical energy are applied. Diguet demonstrated the first photocontrolled and fast manipulation of millimetric liquid drops. All these new approaches are promising for safe manipulation of fragile or toxic samples, and each contributes to a more global strategy for improved exploitation of sunlight energy.

\section{References}

1 Van der Wielen RP, Löwik MR, van den Berg $H$, et al: Serum vitamin D concentrations among elderly people in Europe. Lancet 1995;346:207-210.

-2 Knekt P, Reunanen A, Järvinen R, et al: Antioxidant vitamin intake and coronary mortality in a longitudinal population study. Am J Epidemiol 1994;139:1180-1189.

-3 Yusuf S, Dagenais G, Pogue J, et al: Vitamin E supplementation and cardiovascular events in high-risk patients. The Heart Outcomes Prevention Evaluation Study Investigators. N Engl J Med 2000;342:154-160.
4 Milman U, Blum S, Shapira C, et al: Vitamin E supplementation reduces cardiovascular events in a subgroup of middle-aged individuals with both type 2 diabetes mellitus and the haptoglobin 2-2 genotype: a prospective double-blinded clinical trial. Arterioscler Thromb Vasc Biol 2008;28:341-347.

5 Weber P, Bendich A, Machlin LJ: Vitamin E and human health: rationale for determining recommended intake levels. Nutrition 1997 13:450-460.

6 Moshfegh AZ, Goldman J, Cleveland L: What we eat in America, NHANES 20012002: usual nutrient intakes from food compared to dietary reference intakes. United States Department of Agriculture, Agricultural Research Service, 2005.
7 Breyer F, Costa-Font J, Felder S: Ageing, health, and health care. Oxf Rev Econ Policy 2010;26:674-690.

$>8$ Corpet A, De Koning L, Toedling J, et al: Asf1b, the necessary Asf1 isoform for proliferation, is predictive of outcome in breast cancer. EMBO J 2011;30:480-493.

-9 Radl S, Khinast JG: Prediction of mass transfer coefficients in non-Newtonian fermentation media using first-principles methods. Biotechnol Bioeng 2007;97:1329-1334.

$>10$ Diguet A, Mani NK, Groffroy M, et al: Photosensitive surfactants with various hydrophobic tail lengths for the photocontrol of genomic DNA conformation with improved efficiency. Chemistry 2010;16:11890-11896. 